\title{
Effectiveness and safety of CyberKnife radiosurgery in treatment of trigeminalgia - experiences of Polish neurological and oncological centres
}

\author{
Wiesław Bal ${ }^{1}$, Beata Łabuz-Roszak ${ }^{2,3}$, Rafał Tarnawski $^{4}$, Anetta Lasek-Bal ${ }^{2}$ \\ ${ }^{1}$ Department of Outpatient Chemotherapy, Maria Skłodowska-Curie Memorial Cancer Centre and Institute of Oncology, Gliwice, \\ Poland \\ ${ }^{2}$ Department of Neurology, Medical University of Silesia, School of Health Sciences, Katowice, Poland \\ ${ }^{3}$ Department of Basic Medical Sciences, School of Health Sciences in Bytom, Medical University of Silesia, Katowice, Poland \\ ${ }^{4}$ III Department of Radio- and Chemotherapy, Maria Skłodowska-Curie Memorial Cancer Centre and Institute of Oncology, Gliwice, Poland
}

\begin{abstract}
Trigeminal neuralgia (TN) is one of the most common cranial neuropathies. Pathologies located alongside the long nerve can also cause its mechanical compression or secondary involvement in the inflammatory process, and thus cause pain.

TN is characterised by severe paroxysmal unilateral facial pain in the innervation area of branches I-III of the nerve $\mathrm{V}$ when provoked by light touch or slight movement. Multiple therapeutic methods are available, but most of them yield unsatisfactory results. According to guidelines (AAN and EFNS) the first-line therapy in trigeminalgia is carbamazepine/oxcarbazepine, and if there is a poor response - surgical treatment [1]. The array of surgical options includes percutaneous retrogasserian glycerol injection, radiofrequency thermocoagulation, balloon decompression, thermal rhizotomy, and stereotactic radiosurgery [2-4]. This paper presents our own experiences with CyberKnife (CK), a new type of radiosurgical (RS) treatment of 64 TN patients.

Conclusions CyberKnife radiotherapy is characterised by high efficacy in $80 \%$ of patients with trigeminalgia, minimal invasiveness, and subsiding mild side effects. Radioablation of nerve $V$ root in patients with neuralgia allows us to entirely stop antiepileptic therapy or reduce its doses, which in turn reduces the risk of potential side effects. CyberKnife can be a therapeutic option in those patients who have been offered ineffective therapies, or treatments with limited efficacy, and/or in older patients with comorbidities.
\end{abstract}

Key words: trigeminal neuralgia, CyberKnife, radiosurgery

(Neurol Neurochir Pol 2020; 54 (1): 28-32)

Trigeminal neuralgia (TN) is one of the most common types of cranial nerve pain. Idiopathic $\mathrm{TN}$ is commonly related to patients aged 40-60 but it can occur earlier. Pathologies located alongside the long nerve can also cause its mechanical compression or secondary involvement in the inflammatory process, and thus cause pain. The pathways of the trigeminal nerve include the cervical spine, brainstem, nerve root and the three divisions V1-V3. TN is characterised by severe paroxysmal unilateral facial pain in the innervation area of branches I-III of the nerve $\mathrm{V}$ when provoked by light touch or slight movement. The molecular basis for classic trigeminal pain is uncertain. Its pathophysiology has been modelled by the 'ignition theory', which posits that injured trigeminal neurons fire spontaneously and have a lower threshold for evoked afterdischarges, leading to paroxysmal pain. Voltage-gated sodium channels are responsible for the generation and conduction of action potentials in the peripheral nociceptive neuronal pathway. The intensity of pain and its recurrent nature affect the daily functioning of patients while worsening their quality of life, causing anxiety and often depressive states. Multiple therapeutic methods are available, but most of them yield unsatisfactory results. According to the European Academy 
of Neurology (EAN), the first line of therapy in trigeminalgia is carbamazepine/oxcarbazepine, and this remains the most effective medication especially in the early stages of TN [1].

However, if these drugs become ineffective, or result in poor tolerability, then other drugs must be considered. Based on low to very low quality of evidence, lamotrigine, gabapentin, botulinum toxin type A, pregabalin, baclofen and phenytoin may be used either as monotherapy or combined with carbamazepine or oxcarbazepine when first-line drugs fail in terms of either efficacy or tolerability. If the pharmacotherapy is of no or minimal benefit, surgery should be considered [1]. The array of surgical options includes percutaneous retrogasserian glycerol injection, radiofrequency thermocoagulation, balloon decompression, thermal rhizotomy and stereotactic radiosurgery [2-4]. Unfortunately, evidence regarding the efficacy of interventional procedures is limited. The interest in using radioablation to control pain in patients with TN has been growing in recent years.

This paper presents our own experiences with CyberKnife (CK), a new type of radiosurgical (RS) treatment of $64 \mathrm{TN}$ patients.

\section{Patients and methods}

This retrospective study enrolled patients consulted/ treated because of trigeminal neuralgia in the Department/ Outpatient Clinic of the Neurology Medical University of Silesia in Katowice-Ochojec in the period 2014-2019. Only patients with $\mathrm{TN}$ which did not result from nerve compression by a pathological intracranial mass qualified for being treated with CK. All patients had a brain MRI and brain angio-MRI performed as part of an earlier causative diagnosis of TN and the enrollment process. The Fazekas scale was used to evaluate lesions found in brain MRI unrelated to TN [5]. The inclusion criteria were: disease duration of at least 12-months, previous ineffective therapy and/or its unacceptable side effects. BNI (Barrow Neurological Institute) Pain Intensity Score was used to assess the severity of pain [6]. The patients with at least a IIIb score were included (the assessment covered a recent six-month period). Data related to comorbidities and concurrent therapies was obtained.

The demographic and clinical characteristics of the patients are set out in Table 1.

The CyberKnife radiosurgery was undertaken at the Maria Skłodowska-Curie Memorial Cancer Centre and Institute of Oncology in Gliwice, Poland. Patients were irradiated using the CyberKnife ${ }^{\varpi}$ which is a frameless, image-guided robotic radiosurgery system. It consists of a $6 \mathrm{MV}$ linear accelerator mounted on a robotic arm connected to a robotic couch and a tracking system allowing for correction of the patient's position during radiotherapy. The treatment plans were prepared using CT-MRI fusion in the Multiplan system. 50 patients received $60 \mathrm{~Gy}$ in a single fraction and all the others received 70 Gy in two fractions (35 Gy per fraction) delivered every

\begin{tabular}{|c|c|}
\hline Parameter & n (\%) \\
\hline Age, mean [min-max] & $60.37 \pm 9.4[34-73]$ \\
\hline Patients aged $\leq 65, \mathrm{n}(\%)$ & $22(34.3)$ \\
\hline Sex, F/M & $30 / 34$ \\
\hline Time from onset, years, mean [min-max] & $4.68[1-20]$ \\
\hline NV compression, $\mathrm{n}(\%)$ & $29(45.3)$ \\
\hline Pain laterality right/left & $34 / 30$ \\
\hline \multicolumn{2}{|l|}{ Pain distribution } \\
\hline$\cdot \mathrm{V} 1$ & $2(3.1)$ \\
\hline$\cdot \mathrm{V} 2$ & $15(26.6)$ \\
\hline - V3 & $40(62.5)$ \\
\hline$\cdot \mathrm{V} 2+\mathrm{V} 3$ & $7(10.9)$ \\
\hline Previous antiepileptic therapy, $\mathrm{n}(\%)$ & $56(87.5)$ \\
\hline - $\mathrm{CBZ}$ & $40(62.5)$ \\
\hline - GBP & $20(31.2)$ \\
\hline - LTG & $10(15.6)$ \\
\hline - $P G B$ & $12(18.7)$ \\
\hline - TMD & $7(10.9)$ \\
\hline - NLPZ & $4(6.2)$ \\
\hline - ADS & $8(12.5)$ \\
\hline - STR & $6(9.3)$ \\
\hline Polytherapy, n (\%) & $20(31.2)$ \\
\hline \multicolumn{2}{|l|}{ BNI score before CK, $n(\%)$} \\
\hline · IIIb & $4(6.2)$ \\
\hline . IV & $35(54.7)$ \\
\hline$\cdot \mathrm{V}$ & $25(39.0)$ \\
\hline \multicolumn{2}{|l|}{ Comorbidities, n (\%) } \\
\hline - $\mathrm{AH}$ & $27(42.2)$ \\
\hline - DM & $11(17.2)$ \\
\hline$\cdot$ LD & $42(65.6)$ \\
\hline - MS & $3(4.7)$ \\
\hline - cancer & $9(14.1)$ \\
\hline intracranial (asymptomatic meningioma) & $3(4.7)$ \\
\hline $\begin{array}{l}\text { NV conflict (based on angio-MRl of head), } \\
n(\%)\end{array}$ & $29(45.3)$ \\
\hline Other abnormalities in head MRI, $\mathrm{n}(\%)$ & $14(21.9)$ \\
\hline - MS & $3(4.7)$ \\
\hline - CVD (1-3/Fazekas) & $9(14.1)$ \\
\hline
\end{tabular}

$\mathrm{N}$ - number of patients, $\mathrm{F}$ - female, $\mathrm{M}$ - male, CBZ - carbamazepine, GBP - gabapentin, LTG - lamotrigine, PGB - pregabalin, TMD - tramadol, NSAIDs - nonsteroidal anti-inflammatory drugs, ADS - antidepressant, STR - steroids, BNIS - Barrow Neurological Institute Score, NV - neurovascular, AH - atrial hypertension, DM - diabetes mellitus, LD - lipid disorders, MS - multiple sclerosis, CVD - cerebrovascular disease

other day. The Clinical Target Volume (CTV) consisted of the nerve V root. To create the Planning Target Volume (PTV), a $1 \mathrm{~mm}$ margin in all directions was applied to the CTV (Fig.1). 


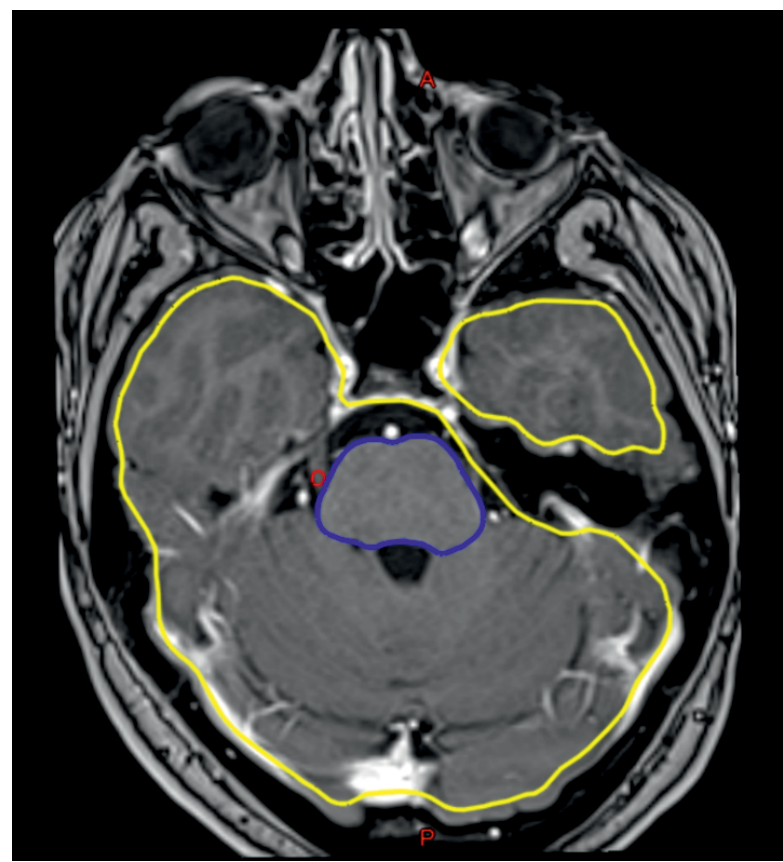

Figure 1. MRI-guided location of the right Gasserian ganglion. The red arrow shows the right Gasserian ganglion planned for radiosurgical ablation

Repeated evaluation by BNI was performed in all patients after three and six months, and in 53 patients after 12 months from CK. Logistic regression analysis was carried out to identify the potential independent prognostic factors favourable in terms of prognosis (BNI $\geq \mathrm{IIIb}$ ); these were chosen from the following: age, sex, duration of disease, symptom laterality and previous treatment. We compared the effects of CK between patients with neurovascular compression vs patients with TN of another aetiology.

\section{Results}

Sixty six patients were initially included for treatment, but two of them were excluded due to comorbid connective tissue disease (lupus erythematosus and facial hemiatrophy). Eventually, 64 patients, including 30 women, made up the study; mean age was 60.37 years. In $29(43.51 \%)$ patients, TN was due to compression (by contiguous vessel), as confirmed on the basis of neurodiagnostic procedures. Neuralgia most often occurred on the right side of the face, in the area of the second and third branches of nerve V. Fifty-six patients (87.5\%) used chronic antiepileptic therapy to control their pain, most often with the use of carbamazepine; of these, 20 patients used more than one drug concurrently. During previous therapy, four patients had obtained satisfactory pain control (IIIb/BNI); however, the polytherapy applied caused unacceptable side effects (in one: diplopia and dizziness, in one: diplopia, in two: skin lesions and dizziness/disequilibrium). Others reported ineffective therapy or effects that fell below expectations (IV-V/BNI).
The effect of therapy assessed according to BNI was as follows.

At three months after CK, 39 patients rated their pain as grade IV or V according to BNI; 51 patients assessed their pain at six months. Thirteen patients in that period did not achieve any beneficial effect, of whom six reported pain at the same level as before CK (four were treated for depression, two used anxiolytic therapy). At six months following CK, 16 patients continued the use of their antiepileptic drugs. So far, 53 patients (82.81\%) have completed at least a 1-year follow-up, and in that group 43 patients (81\%) still assess their pain management as very good (pain absent or occasional, BNI score I or II). Ten (18.86\%) patients permanently or periodically take AED (monotherapy); in each case, the therapy is taken at a lower daily dose than before CK. The clinical effect (BNI score) following CK is presented in Table 2.

Of the 53 patients who completed the 12 months follow-up after $\mathrm{CK}$, there were 24 patients with TN compression aetiology. Of these, 19 patients (79.16\%) reported pain control to be very good (I or II BNI score); four patients still needed AED at a reduced dosage. The percentage of patients with very good results was similar to that observed among patients without evidence of nerve compression -29 patients completed one year including $24(82.75 \%)$ with effect at level I or II BNI score (six patients with AED).

Seven patients underwent CK twice, and four of them improved in terms of their well-being. The reason for the repeated CK session was the lack of clinical efficacy of CK. The period between CK therapies was at least three months.

Side effects related to CK: increased neuralgia severity in two patients for one month after CK, numbness and/or loss of superficial facial cutaneous sensation in a total of 17 patients (26.56\%); this was most severe in the first three months after $\mathrm{CK}$. In each case, the patients assessed the symptoms as being reduced. The pain subsided after repeated CK. The side effects of $\mathrm{CK}$ are presented in Table 2.

Table 2. Clinical parameters following CK

$\begin{array}{lc}\text { BNI IV-V, } 3 \text { months following CK, } n \text { (\%) } & 39(60.9) \\ \text { BNI IV-V, } 6 \text { months following CK, } n \text { (\%) } & 51(79.6) \\ \text { BNI IV-V, } 12 \text { months following CK*, } n(\%) & 43(81.0) \\ \text { AED } 6 \text { months following CK, } n(\%) & 16(25.0) \\ \text { Reduced AED dose, } n(\%) & 16(25.0) \\ \text { Repeated CK therapy, } n(\%) & 7(10.9) \\ \text { Side effects } & \\ \text { - Worsening of trigeminalgia } & 2(3.12) \\ \text { - Loss of sensation within innervation of nerve V } & 15(23.43) \\ \text { - Numbness of face } & 5(7.8) \\ \text { Observation time following CK, years } & 1-5\end{array}$

*Group of 53 patients, BNI - Barrow Neurological Institute Score, CK - CyberKnife, AED - antiepileptic drug 


\section{Discussion}

The most important findings in our study involving 64 patients are as follows: $\mathrm{CK}$ is an effective and well-tolerated method of neuralgia treatment regardless of $\mathrm{TN}$ aetiology, and can be repeated to improve its efficacy without the risk of increased side effects.

It can be a therapeutic option for older patients with multiple comorbidities and/or contraindications for alternative treatment methods (neurosurgery and chronic pharmacological therapy). Sixty-one percent of patients, regardless of the aetiology of disease, obtained a beneficial effect from the therapy over at least three months of follow-up. This percentage increased to $80 \%$ at six months following therapy.

Among our patients, the most common was idiopathic TN or with a confirmed neurovascular conflict (neurovascular compression). Three of them were diagnosed with multiple sclerosis, which suggests an underlying demyelination process in these cases. According to epidemiological data, essential (classic) TN most often occurs as a result of nerve compression by a nearby vessel [7]. The pathophysiological mechanism of compression is not entirely known. Structural studies have revealed axonal loss and demyelination or dysmyelination associated with compression. The involvement of genetic factors is probable. Repetitive results were obtained suggesting possible mutation in voltage-gated sodium channel $\mathrm{NaV}$ 1.6, $\mathrm{NaV} 1.7, \mathrm{NaV} 1.8$, and $\mathrm{NaV} 1.9$ in TN patients [8, 9]. The role of voltage-gated sodium channels in TN is compatible with an appropriate response to carbamazepine and other sodium channel blockers. The clinical spectrum of mutation effects includes trigeminalgia, migraine pains, and epileptic encephalopathy $[9,10]$. Independently, nerve $\mathrm{V}$ microinjury may result from vascular pulsation [11]. Segmental myelin damage may be associated with receptor reorganisation and ectopic impulse generation. The coexistence of several mechanisms of nerve damage in one patient cannot be excluded, which may justify combining non-pharmacological and pharmacological methods in some patients. A lasting effect of therapy most often occurred after 6-8 weeks from CK. In $11 \%$ of patients, we used CK twice to achieve the intended therapeutic effect. In accordance with the reports by other authors, the analgesic effect of radiosurgery (gamma knife) was observed at 1-11 weeks following treatment [12-15]. We observed an increase in symptoms within the first month after CK in two patients; these eventually resolved after another radioablation. This can be explained by an acute inflammatory process only partially involving the damaged root. An optimal cumulative safe radiation dose has not been established; some suggest that it should be $<130 \mathrm{~Gy}$, while others propose $>150 \mathrm{~Gy}[16$, 17]. We found no significant differences in the effectiveness between low and high doses; however, some reports contradict these observations [18].

Our observations also indicate an improvement in the effectiveness after a repeated CK session. A particular role may be played by individual radiation sensitivity, which could explain the different effects of therapy and the need to repeat it in some patients. It is worth stressing that depression was more frequent among those patients who required retreatment and/ or who had been administered a previous anxiolytic therapy. None of the parameters under study related to the patient, and the therapeutic technique turned out to be an independent prognostic factor for CK. Similar to the experiences of other authors, the only predictor of a favourable response to therapy was the compression aetiology of TN $[19,20]$.

In the present study, $25 \%$ of patients continued antiepileptic therapy at six months after CK; however, in each case the dose was reduced in relation to the initiation baseline. The percentage of patients requiring continued antiepileptic therapy decreased to $19 \%$ after 12 months following CK.

The most common side effects were numbness and loss of facial sensation in the area of nerve $V$ activity (reported by $26 \%$ of all patients). Our observations in this respect are consistent with those made by other authors [21-23]. Two patients showed an increase in trigeminalgia which was managed with the use of a repeated CK session. In two individuals with connective tissue disease, we did not decide to perform $\mathrm{CK}$, so as not to provoke adverse consequences of the inflammatory process accompanying CK. There are no experiences related to patients with connective tissue diseases. The known contraindications for $\mathrm{CK}$ are consistent with the contraindication for MRI. Before CK, a brain MRI is performed to determine the area for CK-ablation.

Along with the increase in life expectancy and the proportion of elderly patients in the global population, the number of patients with $\mathrm{TN}$ has been increasing.

For these reasons, and the insufficient efficacy of existing therapies, there is a need for new minimally invasive, safe and effective methods of neuropathic pain control. Chronic antiepileptic therapy exposes older patients, who often have other comorbidities, to its side effects. The use of CK seems to be a beneficial therapeutic modality, particularly in this group of patients. CK can be also an alternative to other methods of therapy $[1-4,24]$. Further research is needed to confirm the parameters presented currently in relation to the efficacy and safety of radiosurgery in the treatment of TN.

\section{Limitations}

There were two limitations in our study: its retrospective nature, and a relatively small number of patients.

\section{Conclusions}

CyberKnife radiotherapy is characterised by high efficacy in $80 \%$ of patients with trigeminalgia, minimal invasiveness, and subsiding mild side effects.

Radioablation of nerve $\mathrm{V}$ root in patients with neuralgia allows us to entirely stop antiepileptic therapy or reduce its doses, which in turn reduces the risks of potential side effects. 
CyberKnife can be a therapeutic option in those patients who have been offered ineffective therapies or treatments with limited efficacy and/or in older patients with comorbidities.

\section{References}

1. Bendtsen L, Zakrzewska JM, Abbott J, et al. European Academy of Neurology guideline on trigeminal neuralgia. Eur J Neurol. 2019; 26(6): 831-849, doi: 10.1111/ene.13950, indexed in Pubmed: 30860637.

2. Zakrzewska JM, Akram H. Neurosurgical interventions for the treatment of classical trigeminal neuralgia. Cochrane Database Syst Rev. 2011(9): CD007312, doi: 10.1002/14651858.CD007312.pub2, indexed in Pubmed: 21901707.

3. Sharim J, Lo WL, Kim W, et al. Radiosurgical target distance from the root entry zone in the treatment of trigeminal neuralgia. Pract Radiat Oncol. 2017; 7(4): 221-227, doi: 10.1016/j.prro.2016.12.006, indexed in Pubmed: 28336479.

4. Debono B, Lotterie JA, Sol JC, et al. Dedicated Linear Accelerator Radiosurgery for Classic Trigeminal Neuralgia: A Single-Center Experience with Long-Term Follow-Up. World Neurosurg. 2019; 121: e775-e785, doi: 10.1016/j.wneu.2018.09.222, indexed in Pubmed: 30312817.

5. Zerna C, Yu AYX, Modi J, et al. Association of White Matter Hyperintensities With Short-Term Outcomes in Patients With Minor Cerebrovascular Events. Stroke. 2018; 49(4): 919-923, doi: 10.1161/ STROKEAHA.117.017429, indexed in Pubmed: 29540612.

6. Erdine S, Ozyalcin NS, Cimen A, et al. Comparison of pulsed radiofrequency with conventional radiofrequency in the treatment of idiopathic trigeminal neuralgia. Eur J Pain. 2007; 11(3): 309-313, doi: 10.1016/j.ejpain.2006.04.001, indexed in Pubmed: 16762570.

7. Devor M, Govrin-Lippmann R, Rappaport ZH. Mechanism of trigeminal neuralgia: an ultrastructural analysis of trigeminal root specimens obtained during microvascular decompression surgery. J Neurosurg. 2002; 96(3): 532-543, doi: 10.3171/jns.2002.96.3.0532, indexed in Pubmed: 11883839.

8. Dib-Hajj SD, Geha P, Waxman SG. Sodium channels in pain disorders: pathophysiology and prospects for treatment. Pain. 2017; 158 Suppl 1: S97-S9S107, doi: 10.1097/j.pain.0000000000000854, indexed in Pubmed: 28240647.

9. Mohammed H, Rimondini L, Rocchetti V. Molecular basis of trigeminal nerve disorders and healing. Eur Rev Med Pharmacol Sci. 2018; 22(17): 5755-5764, doi: 10.26355/eurrev_201809_15844, indexed in Pubmed: 30229854.

10. Tanaka BS, Zhao P, Dib-Hajj FB, et al. A gain-of-function mutation in Nav1.6 in a case of trigeminal neuralgia. Mol Med. 2016; 22: 338-348, doi: 10.2119/molmed.2016.00131, indexed in Pubmed: 27496104.

11. Harsha KJ, Kesavadas $\mathrm{C}$, Chinchure $\mathrm{S}$, et al. Imaging of vascular causes of trigeminal neuralgia. J Neuroradiol. 2012; 39(5): 281-289, doi: 10.1016/j.neurad.2012.08.006, indexed in Pubmed: 23177241.

12. Dvorak T, Finn A, Price LL, et al. Retreatment of trigeminal neuralgia with Gamma Knife radiosurgery: is there an appropriate cumulative dose? Clinical article. J Neurosurg. 2009; 111(2): 359-364, doi: 10.3171/2008.11.JNS08770, indexed in Pubmed: 19326978.

13. Herman J, Petit J, Amin P, et al. Repeat gamma knife radiosurgery for refractory or recurrent trigeminal neuralgia: treatment outcomes and quality-of-life assessment. International Journal of Radiation Oncology*Biology*Physics. 2004; 59(1): 112-116, doi: 10.1016/j. ijrobp.2003.10.041.

14. Kimball BY, Sorenson JM, Cunningham D. Repeat Gamma Knife surgery for trigeminal neuralgia: long-term results. J Neurosurg. 2010; 113 Suppl: 178-183, doi: 10.3171/2010.8.GKS101075, indexed in Pubmed: 21121800.

15. Pollock BE, Schoeberl KA. Prospective comparison of posterior fossa exploration and stereotactic radiosurgery dorsal root entry zone target as primary surgery for patients with idiopathic trigeminal neuralgia. Neurosurgery. 2010; 67(3): 633-8; discussion 638, doi: 10.1227/01. NEU.0000377861.14650.98, indexed in Pubmed: 20647962.

16. Urgosik D, Liscak R, Novotny J, et al. Treatment of essential trigeminal neuralgia with gamma knife surgery. J Neurosurg. 2005; 102 Suppl: 29-33, doi: 10.3171/jns.2005.102.s_supplement.0029, indexed in Pubmed: 15662776.

17. Hasegawa T, Kondziolka D, Spiro R, et al. Repeat Radiosurgery for Refractory Trigeminal Neuralgia. Neurosurgery. 2002; 50(3): 494-502, doi: 10.1227/00006123-200203000-00012.

18. Park $\mathrm{KJ}$, Kondziolka $\mathrm{D}$, Berkowitz $\mathrm{O}$, et al. Repeat gamma knife radiosurgery for trigeminal neuralgia. Neurosurgery. 2012; 70(2): 295-305; discussion 305, doi: 10.1227/NEU.0b013e318230218e, indexed in Pubmed: 21811188.

19. Brisman R, Khandji AG, Mooij RBM. Trigeminal Nerve-Blood Vessel Relationship as Revealed by High-resolution Magnetic Resonance Imaging and Its Effect on Pain Relief after Gamma Knife Radiosurgery for Trigeminal Neuralgia. Neurosurgery. 2002; 50(6): 1261-6, discussion 1266, doi: 10.1097/00006123-200206000-00015, indexed in Pubmed: 12015844.

20. Erbay SH, Bhadelia RA, Riesenburger R, et al. Association between neurovascular contact on MRI and response to gamma knife radiosurgery in trigeminal neuralgia. Neuroradiology. 2006; 48(1): 26-30, doi: 10.1007/s00234-005-0008-5, indexed in Pubmed: 16237547.

21. Jia Y, Pan Y, Ren H, et al. Effectiveness and Safety of High-Voltage Pulsed Radiofrequency to Treat Patients with Primary Trigeminal Neuralgia: A Multicenter, Randomized, Double-Blind, Controlled Study Protocol. Pain Physician. 2018; 21(5): 469-481, indexed in Pubmed: 30282391.

22. Taich ZJ, Goetsch SJ, Monaco E, et al. Stereotactic Radiosurgery Treatment of Trigeminal Neuralgia: Clinical Outcomes and Prognostic Factors. World Neurosurg. 2016; 90: 604-612.e11, doi: 10.1016/j. wneu.2016.02.067, indexed in Pubmed: 26915701.

23. Baschnagel AM, Cartier JL, Dreyer J, et al. Trigeminal neuralgia pain relief after gamma knife stereotactic radiosurgery. Clin Neurol Neurosurg. 2014; 117: 107-111, doi: 10.1016/j.clineuro.2013.12.003, indexed in Pubmed: 24438815.

24. Ostrowski H, Roszak J, Komisarek O. Botulinum toxin type A as an alternative way to treat trigeminal neuralgia: a systematic review. Neurol Neurochir Pol. 2019; 53(5): 327-334, doi: 10.5603/PJNNS. a2019.0030, indexed in Pubmed: 31397877. 